\title{
Morrer no domicílio: fatores associados à satisfação da preferência do doente
}

Rita Carvalho Cunha Ferreira, ${ }^{1,2}$ Manuel Luís Capelas ${ }^{2,3}$

\section{RESUMO}

Introdução: A importância do local de morte tem sido estudada e valorizada em cuidados paliativos. Em Portugal parece que a maioria dos doentes prefere morrer no domicílio, embora a sua preferência não seja satisfeita.

Objetivos: Identificar fatores associados à satisfação da preferência de morte no domicílio entre doentes acompanhados em cuidados paliativos em Portugal.

Métodos: Estudo epidemiológico, observacional, transversal e analítico, em doentes acompanhados durante 2017 nas equipas de cuidados paliativos que responderam a um estudo de maiores dimensões do Observatório Português de Cuidados Paliativos. Foram selecionadas variáveis independentes de caracterização demográfica, social, clínica e de cuidados de saúde. Para análise bivariada foram utilizados os testes $\chi^{2}$, Mann-Whitney e modelo de regressão logística binária. Para análise multivariada foi utilizado o modelo de regressão logística com base no método Forward, baseado no teste de Wald.

Resultados: Obtivemos uma taxa de resposta das instituições de 9,8\%, com inclusão de 68 doentes, dos quais $52,9 \%$ do género masculino e com mediana de 78,5 anos (ampl/Q=19). A satisfação da preferência de morte no domicílio verificou-se em $32,3 \%(k=0,26)$ e associou-se de forma estatisticamente significativa a alguns fatores, nomeadamente à residência nos Açores $(O R=3,79)$, à residência numa área rural $(O R=10,00)$, à duração do seguimento desde a admissão numa equipa até à morte $(\leq 30$ dias, $O R=0,15)$ e ao facto de o doente ser acompanhado por uma equipa comunitária de suporte em cuidados paliativos $(\mathrm{OR}=9,00)$.

Conclusões: O valor de satisfação da preferência de morte no domicílio obtido foi apenas razoável, encontrando-se entre os valores mais baixos da literatura. Foi identificada uma relação com o local de residência, o tempo de seguimento em cuidados paliativos e o acompanhamento por equipas comunitárias especializadas. Estes fatores facilitadores vão ao encontro dos achados em estudos internacionais e poderão orientar a definição de estratégias adequadas à realidade da população portuguesa.

Palavras-chave: Cuidados paliativos; Cuidados em fim de vida; Preferências do doente; Local de morte; Morte no domicílio.

\section{INTRODUÇÃO}

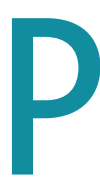

retender respeitar as preferências dos doentes, nos cuidados em fim de vida, implica ter em mente também a preferência do local de morte, assim como reunir esforços de forma que esta possa ser cumprida. De facto, a importância do local de morte tem sido estudada e valorizada pelos doentes, ${ }^{1}$ parecendo inclusive existir uma forte associação entre a ocorrência da morte no local escolhido e a sa-

1. Equipa comunitária de suporte em cuidados paliativos, ACeS Lisboa Ocidental e Oeiras. Oeiras, Portugal.

2. Observatório Português dos Cuidados Paliativos, Instituto de Ciências da Saúde, Universidade Católica Portuguesa. Lisboa, Portugal.

3. Centro de Investigação Interdisciplinar em Saúde, Instituto de Ciências da Saúde, Universidade Católica Portuguesa. Lisboa, Portugal. tisfação com os cuidados em fim de vida por parte dos familiares. $^{2}$

Um estudo do Observatório Português dos Cuidados Paliativos (OPCP) revelou que $69 \%$ dos doentes preferia morrer no domicilio (próprio ou de familiares ou amigos). ${ }^{3}$ Outros estudos anteriores, levados a cabo na população geral, apontavam para valores um pouco inferiores. $^{4-5}$

No entanto, apesar da preferência pela morte no domicílio parecer ser predominante, a satisfação deste desejo atinge-se apenas em $26 \%$ dos doentes, quando se refere ao domicílio do próprio, e $55 \%$ quando se refere ao domicílio de familiares ou amigos. ${ }^{3} \mathrm{O}$ desfasamento entre desejo e realidade é confirmado também através 
das estatísticas nacionais, que revelam que a morte no domicílio atinge apenas os 26\%. ${ }^{6}$ Neste âmbito, existem inclusive previsões para 2030 que revelam que, caso não haja intervenção em Portugal, a proporção de mortes no hospital poderá alcançar pelo menos $75 \% .^{7}$ Para além de ser incomportável para um sistema de saúde, este cenário alerta para um local de morte que caminhará em sentido oposto à preferência dos doentes.

Apesar de não serem conhecidos no nosso país, na literatura internacional foram já identificados, com alguma heterogeneidade, vários fatores que poderão facilitar a morte no domicílio entre os doentes que assim o desejam. Para além dos fatores relacionados com a doença e dos fatores relacionados com o indivíduo, ${ }^{8-10}$ foram também identificados fatores ambientais, onde se destacam, como fatores facilitadores, as visitas domiciliárias de enfermagem, do médico de família e da equipa especializada em cuidados paliativos, ${ }^{8-11}$ assim como o facto de o doente não se encontrar hospitalizado nos últimos três meses de vida, sendo cuidado no domicílio, e o facto de o doente discutir o tema do local de morte com os profissionais de saúde. ${ }^{8,11}$

O objetivo do presente trabalho foi então identificar fatores associados à satisfação da preferência de morte no domicílio entre os doentes acompanhados em cuidados paliativos em Portugal.

\section{MÉTODOS}

O presente estudo foi baseado em dados preliminares gerados por uma investigação abrangente do OPCP, denominada Cuidados Paliativos em Portugal: CARACTERIZAÇÃO DE DOENTES REFERENCIADOS E ADMITIDOS, EQUIPAS, AVALIAÇÃO DE INDICADORES DE QUALIDADE, EFETIVIDADE DO CONTROLO SINTOMÁTICO E LOCAL DE MORTE, ${ }^{12}$ na qual colaboraram os autores do presente estudo. Tratou-se de um estudo epidemiológico, observacional, transversal e analítico, recorrendo ao método quantitativo.

A população foi composta pelos doentes que receberam cuidados paliativos em Portugal durante o ano de 2017, estando acessíveis através das equipas especializadas de cuidados paliativos registadas em fontes oficiais em Portugal Continental e regiões autónomas, no início desse ano. As equipas foram contactadas por email, solicitando-se os dados dos doentes acompanhados. Não tendo sido possível o envio dos dados de todos os doentes e de todas as equipas, ficou assim, con- trariamente ao previsto, constituída uma amostra acidental de participantes no estudo. Como critérios de inclusão selecionou-se a idade maior ou igual a 18 anos e os doentes encontrarem-se cognitivamente capazes, segundo avaliação da equipa prestadora de cuidados. Foram também critérios de inclusão os doentes desejarem morrer no domicílio (próprio ou de familiares ou amigos) e apresentarem o local de morte real registado.

Os dados foram colhidos numa base de dados em Microsoft Excel $₫$ desenvolvida para o efeito, preenchida pelos profissionais prestadores de cuidados paliativos através da consulta dos processos clínicos. Quanto às informações relativas ao cuidador informal do doente, os dados foram colhidos através do autopreenchimento de um questionário e posteriormente registados em Microsoft Excel®. De forma a garantir o anonimato e evitar a duplicação de casos foi utilizado um código para cada doente.

A realização do estudo foi autorizada pelos Conselhos de Administração e obteve parecer favorável das Comissões de Ética para a Saúde das instituições participantes, assim como autorização da Comissão Nacional de Proteção de Dados. De forma a obter o consentimento do doente e do seu cuidador foram solicitados os consentimentos expressos dos próprios ou dos seus representantes legais através de formulário.

Foram selecionadas variáveis independentes de caracterização demográfica (idade; género), caracterização social (estado civil; nível de instrução; local de residência, colhido sob a forma de freguesia e localidade, mas analisado consoante a região NUTS II de Portugal e consoante a tipologia de áreas urbanas adotada pelo Instituto Nacional de Estatística), ${ }^{13}$ caracterização clínica (diagnóstico clínico que motivava o acompanhamento em cuidados paliativos, agrupando em oncológico e não oncológico; sensação de bem-estar funcional no momento da admissão, através da aplicação do questionário FACIT-Pal, ${ }^{14}$ com validação linguística para a língua portuguesa ${ }^{15} \mathrm{e}$ após autorização da entidade detentora da propriedade intelectual) e, finalmente, variáveis de caracterização dos cuidados de saúde (tipologia de equipas de cuidados paliativos onde o doente era acompanhado; duração do seguimento na equipa, em dias; existência de cuidador de referência, considerado pela equipa e pelo doente, e a sua situação laboral, o seu grau de parentesco com o doente e o 
tempo despendido como cuidador por semana, medido em horas). De forma a ultrapassar um possível viés de aferição, o questionário FACIT-Pal foi aplicado entre as $0 \mathrm{~h}$ e as $48 \mathrm{~h}$ para permitir alguma estabilização do doente e a equipa prestadora foi instruída, em reunião prévia, para não interpretar as possíveis hipóteses de resposta na escala deste questionário.

Os dados foram analisados com recurso a medidas epidemiológicas, estatística descritiva e estatística analítica, com o apoio dos seguintes softwares: Microsoft Excel®, IBM SPSS ${ }^{\circledR}$ v. 23, EpiDat ${ }^{\circledR}$ v. 3.1. A análise da normalidade das variáveis contínuas foi realizada com recurso ao teste Kolmogorov-Smirnov. Para analisar a variável dependente considerou-se o domicílio num sentido abrangente, englobando o domicílio do próprio e o domicílio de familiares ou amigos, pois, apesar de se saber que para um indivíduo desejar morrer em sua casa é diferente de desejar morrer em casa de um familiar ou de um amigo, ${ }^{16}$ numa perspetiva de distribuição e gestão de cuidados fez sentido esta visão. Para análise bivariada foram utilizados os testes $\chi^{2}$ e Mann-Whitney. Uma vez que as variáveis foram avaliadas de forma independente, os dados omissos foram assumidos como «não resposta», não sendo considerados esses doentes na análise bivariada. Na aplicação do questionário FACIT-Pal, as regras do cálculo do score já contemplam a possibilidade de dados omissos, pelo que estes não interferiram na variável do bem-estar funcional. Foi utilizado o modelo de regressão logística binária também para a análise bivariada, assim como para análise multivariada, com base no método Forward baseado no teste de Wald, com inclusão das variáveis que, na análise bivariada, obtiveram associação estatisticamente significativa com a variável dependente.

\section{RESULTADOS}

Entre as 61 instituições de saúde listadas e contactadas no estudo do OPCP, apenas seis enviaram dados (taxa de resposta das instituições de 9,8\%), apresentando a seguinte tipologia: três equipas comunitárias de suporte em cuidados paliativos (ECSCP), uma equipa intra-hospitalar de suporte em cuidados paliativos (EIHSCP) e duas unidades de cuidados paliativos (UCP).

Após exclusão de um doente repetido obtiveram-se 376 doentes cognitivamente capazes e com idade maior ou igual a 18 anos. Entre estes, 197 não foram incluídos no estudo por desejarem morrer noutro local diferente do domicílio ou por não responderem em relação ao desejo do local de morte. Entre os 179 restantes, 111 não foram incluídos por não apresentarem o local de morte registado. Resultou, assim, uma amostra de 68 doentes, sendo $52,9 \%$ do género masculino (Tabela 1 ). A idade apresentou uma distribuição não normal (KS (68) $=0,127, p<0,05)$, com $72,1 \%$ dos doentes com idade compreendida entre os 60 e 89 anos. A mediana da idade foi de 78,5 anos, distribuindo-se entre os 42 e os 94 anos (ampIIQ=19). Em relação ao estado civil, 52,9\% dos doentes era casado (com ou sem registo). Quanto ao nível de instrução, 91,0\% apresentava nenhuma escolaridade ou apenas ensino básico. No que se refere à região de Portugal, 51,5\% dos doentes residia no arquipélago dos Açores, 32,4\% na região de Lisboa e nenhum doente na região do Algarve e arquipélago da Madeira. A residência em áreas predominantemente urbanas verificou-se em 72,1\% dos doentes (Tabela 1 ).

Em relação à caracterização clínica, 79,4\% apresentava uma doença oncológica que motivava o acompanhamento em cuidados paliativos (Tabela 1). Também relativo à caracterização clínica foi avaliado o bem-estar funcional na admissão, registado apenas em 11 doentes, verificando-se uma distribuição não normal do resultado da avaliação ( $\mathrm{SW}(11)=0,81, p<0,05)$, entre 6 e 14 pontos, com uma mediana de 7 ( $a m p I I Q=6,8)$.

No que se refere aos cuidados de saúde, $55,9 \%$ dos doentes era cuidado no domicílio e $36,8 \%$ numa UCP (Tabela 1). Entre os 50 doentes com data de admissão na equipa e data de óbito registadas verificou-se uma distribuição não normal da duração do seguimento na equipa (SW $(50)=0,598, p<0,001$ ), entre 1 e 1.057 dias, com mediana de 35,5 dias (ampIIQ=87).

A presença de cuidador informal verificou-se em $98,5 \%$ dos doentes. Entre os 66 doentes com situação laboral do cuidador registada, $53,0 \%$ era cuidado por uma pessoa inativa e $31,8 \%$ por uma pessoa empregada por conta de outrem. O cuidador informal era filho em 45,5\% dos doentes e cônjuge ou companheiro em 40,9\% (Tabela 1). O tempo despendido como cuidador por semana teve uma distribuição não normal (KS (65) $=0,304, p<0,001)$, com mediana de 49 horas, variando entre 2 e 168 horas por semana ( $\mathrm{am}$ $p I I Q=52,5)$. 


\begin{tabular}{|c|c|}
\hline Característica & $N(\%)$ \\
\hline \multicolumn{2}{|l|}{ Género } \\
\hline Masculino & $36(52,9)$ \\
\hline Feminino & $32(47,1)$ \\
\hline \multicolumn{2}{|l|}{ Faixa etária (anos) } \\
\hline $42-49$ & $3(4,4)$ \\
\hline $50-59$ & $7(10,3)$ \\
\hline $60-69$ & $8(11,8)$ \\
\hline $70-79$ & $17(25,0)$ \\
\hline $80-89$ & $24(35,3)$ \\
\hline $90-94$ & $9(13,2)$ \\
\hline Idade (anos) mediana (ampllQ) & $78,5(19,0)$ \\
\hline \multicolumn{2}{|l|}{ Estado civil } \\
\hline Casado(a) com ou sem registo & $36(52,9)$ \\
\hline Outro & $32(47,1)$ \\
\hline \multicolumn{2}{|l|}{ Nível de instrução $(n=67)$} \\
\hline Nenhum & $17(25,4)$ \\
\hline Básico & $44(65,7)$ \\
\hline Secundário & $5(7,5)$ \\
\hline Especialização tecnológica & 0 \\
\hline Superior & $1(1,5)$ \\
\hline \multicolumn{2}{|l|}{ Local de residência (NUTS II) } \\
\hline Açores & $35(51,5)$ \\
\hline Lisboa & $22(32,4)$ \\
\hline Centro & $7(10,3)$ \\
\hline Norte & $3(4,4)$ \\
\hline Alentejo & $1(1,5)$ \\
\hline \multicolumn{2}{|l|}{ Local de residência (grau de urbanização) } \\
\hline Área predominantemente urbana & $49(72,1)$ \\
\hline Área mediamente urbana & $14(20,6)$ \\
\hline Área predominantemente rural & $5(7,4)$ \\
\hline \multicolumn{2}{|l|}{ Diagnóstico clínico } \\
\hline Oncológico & $54(79,4)$ \\
\hline Não oncológico & $14(20,6)$ \\
\hline $\begin{array}{l}\text { Bem-estar funcional }(n=11) \text { mediana } \\
\text { (amplIQ) }\end{array}$ & $7,0(6,8)$ \\
\hline \multicolumn{2}{|l|}{ Tipologia da equipa de cuidados paliativos } \\
\hline ECSCP & $38(55,9)$ \\
\hline UCP & $25(36,8)$ \\
\hline EIHSCP & $5(7,4)$ \\
\hline
\end{tabular}

\begin{tabular}{|l|r|}
\hline \multicolumn{2}{|l|}{ TABELA 1. (continuação) } \\
Característica & \multicolumn{1}{|c|}{$N(\%)$} \\
\hline Duração do seguimento desde a admissão & $35,5(87,0)$ \\
(dias) ( $n=50)$ mediana (amplIQ) & \\
\hline Existência de cuidador & $67(98,5)$ \\
Sim & $1(1,5)$ \\
$\quad$ Não & \\
\hline Situação laboral do cuidador ( $n=66)$ & $35(53,0)$ \\
Inativo & $21(31,8)$ \\
Empregado por conta de outrem & $8(12,1)$ \\
$\quad$ Empregado por conta própria & $2(3,0)$ \\
Desempregado & $30(45,5)$ \\
\hline Grau de parentesco do cuidador ( $n=66)$ & $27(40,9)$ \\
Filho & $4(6,1)$ \\
Cônjuge/companheiro & $3(4,5)$ \\
Outro & $2(3,0)$ \\
Outro parente & $49,0(52,5)$ \\
Irmão & \\
\hline Tempo despendido como cuidador (horas) \\
( $n=66)$ mediana (ampllQ)
\end{tabular}

Legenda: NUTS = Nomenclatura das unidades territoriais para fins estatísticos; ECSCP = Equipa comunitária de suporte em cuidados paliativos; $\mathrm{UCP}=$ Unidade de cuidados paliativos; ElHSCP = Equipa intra-hospitalar de suporte em cuidados paliativos.

\section{Local de ocorrência de morte}

Quanto ao local em que ocorreu a morte, apenas houve concordância com o desejo em $32,3 \%$ (IC $_{95 \%}$ 20,50-44,21), obtendo-se um valor $k$ de $0,26(p<0,001)$. Quanto aos restantes doentes, 36,8\% ( IC $\left._{95 \%} 24,57-48,96\right)$ morreu numa UCP e 30,9\% ( IC $\left._{95 \%} 19,17-42,60\right)$ num hospital que não UCP (Tabela 2).

\section{Análise bivariada}

De forma a identificar os fatores associados à satisfação da preferência de morte no domicílio foram analisadas diferentes variáveis (Tabela 3 ).

No que se refere a variáveis de caracterização demográfica e de caracterização clínica não se obteve qualquer relação estatisticamente significativa.

Quanto a variáveis de caracterização social foi identificada uma associação estatisticamente significativa com a residência nos Açores vs resto do país $(\mathrm{OR}=3,79$; 


\begin{tabular}{|c|c|c|}
\hline Local de ocorrência de morte & $N$ & $\%$ \\
\hline UCP & 25 & 36,8 \\
\hline Domicílio & 22 & 32,3 \\
\hline Próprio & 16 & 23,5 \\
\hline De familiar ou amigo & 6 & 8,8 \\
\hline Hospital ou outra unidade não UCP & 21 & 30,9 \\
\hline Lar ou residência & 0 & 0,0 \\
\hline
\end{tabular}

Legenda: UCP = Unidade de cuidados paliativos.

$\left.\mathrm{IC}_{95 \%} 1,25-11,46\right)$ e com a residência numa área rural $v s$ área mais urbanizada (OR=10,00; $\left.\mathrm{IC}_{95 \%} 1,05-95,68\right)$.

Em relação às variáveis de caracterização dos cuidados de saúde verificou-se uma associação com a duração do seguimento desde a admissão numa equipa até à morte ( $\leq 30$ dias $v s>30$ dias; $\left.\mathrm{OR}=0,15 ; \mathrm{IC}_{95 \%} 0,03-0,74\right)$ e com o facto de o doente ser cuidado por uma ECSCP vs acompanhamento em tipologias com internamento $\left(\mathrm{OR}=9,00 ; \mathrm{IC}_{95 \%} 2,33-34,78\right)$.

\section{Regressão logística}

Foi realizada uma regressão logística para verificar se existiria modelo preditivo em relação à duração do seguimento desde a admissão até à morte, mas não foi encontrado (OR=1,00; $\left.\mathrm{IC}_{95 \%} 1,000-1,005, p=0,198\right)$.

Foi também efetuada uma regressão logística com o método de Forward, integrando as variáveis independentes com significância estatística na análise bivariada (local de residência por região NUTS II, local de residência por tipologia de área urbana, duração do seguimento desde a admissão numa equipa até à morte, tipologia da equipa de cuidados paliativos onde o doente era acompanhado), resultando um modelo, embora estatisticamente significativo, não suficientemente preditivo porque a percentagem de casos classificados pelo modelo de regressão $(78 \%)$ apenas foi superior em $8 \%$ ao modelo nulo, quando deveria ser no mínimo em $25 \%$. Apenas uma categoria de uma variável independente, o acompanhamento numa ECSCP $v$ s tipologias com internamento, obteve significância estatística com um OR de 13,50 e IC $_{95 \%}$ 3,040-59,960.

\section{DISCUSSÃO}

A amostra obtida apresentou uma ligeira predominância do sexo masculino e uma maior representação da oitava e nona décadas de vida. Esta distribuição vai ao encontro das previsões da OMS, as quais estimam que $52 \%$ dos doentes a necessitar de cuidados paliativos seja do género masculino e $69 \%$ se encontre acima dos 60 anos. $^{17}$

Quanto à preferência de morte no domicílio obteve-se uma satisfação do desejo de apenas 32,3\%, encontrando-se, sem dúvida, entre os valores mais baixos da literatura, ${ }^{8-10,16,18-23} \mathrm{com}$ um valor $\mathrm{k}$ considerado apenas razoável.

No presente estudo foram identificados alguns fatores com associação estatisticamente significativa com a satisfação da preferência de morte no domicílio.

$\mathrm{O}$ facto de um doente residir nos Açores aumentou cerca de quatro vezes a probabilidade de concretizar o desejo de morrer no domicílio quando comparado com os residentes nas restantes regiões.

Também a residência numa área predominantemente rural atuou como fator facilitador da concretização da preferência por morrer no domicílio, aumentando dez vezes essa probabilidade. Esta associação poderá dever-se a motivos culturais e a valores mais tradicionais, como o valor da família e a relação de vizinhança, associados a uma menor disponibilidade ou acessibilidade a hospitais. Neste sentido, ter-se-á de assegurar que a preferência e a ocorrência da morte no domicílio nestes locais são de facto "escolhas pela positiva" e não "escolhas pela negativa", isto é, por não disporem de outra opção. ${ }^{24}$

A relação identificada com o local de residência, quer em termos de região quer em termos de grau de urbanização, encontra-se documentada na literatura, embora não seja relativa à satisfação do desejo, mas à preferência e à ocorrência de morte no domicílio, analisadas de forma separada. ${ }^{4-5,10,23-26}$

A duração do seguimento desde a admissão na equipa até ao óbito também influenciou a probabilidade de satisfação da preferência de morrer no domicílio, sendo que o grupo de doentes com acompanhamento menor ou igual a um mês apresentou uma menor probabilidade de concretizar a sua preferência. Parece então que uma admissão (e, consequentemente, uma referenciação) atempada atuou como fator facilitador. Este 


\begin{tabular}{|c|c|c|c|c|}
\hline Variável & Classe em análise & OR & IC 95\% & Valor $p$ \\
\hline Idade ${ }^{\S}$ & & 1,02 & $0,98-1,06$ & 0,373 \\
\hline Género & Masculino vs. feminino* & 1,86 & $0,67-5,16$ & 0,222 \\
\hline Estado civil & Casado vs. restantes* & 1,01 & $0,36-2,80$ & 0,988 \\
\hline Nível de instrução & Nenhuma/básica vs. restantes* & 1,95 & $0,30-12,75$ & 0,655 \\
\hline \multirow[t]{5}{*}{ Residência (NUTS II) } & Lisboa vs. restantes* & 0,35 & $0,10-1,19$ & 0,084 \\
\hline & Açores vs. restantes* & 3,79 & $1,25-11,46$ & 0,015 \\
\hline & Norte vs. restantes* & 4,50 & $0,39-52,54$ & 0,243 \\
\hline & Centro vs. restantes* & 0,30 & $0,03-2,62$ & 0,41 \\
\hline & Alentejo vs. restantes* & 2,14 & $0,13-35,94$ & 0,55 \\
\hline \multirow[t]{2}{*}{ Residência (grau urbanização) } & APU vs. restantes* & 0,45 & $0,15-1,35$ & 0,150 \\
\hline & APR vs. restantes* & 10,00 & $1,05-95,68$ & 0,035 \\
\hline Diagnóstico clínico* & & 1,99 & $0,49-8,02$ & 0,523 \\
\hline Bem-estar funcional ${ }^{\S}$ & & 1,63 & $0,78-3,38$ & 0,099 \\
\hline Tipologia da equipa & ECSCP vs. restantes* & 9,00 & $2,33-34,78$ & $<0,001$ \\
\hline \multirow[t]{2}{*}{ Duração do seguimento desde a admissão } & $\S$ & 1,00 & $1,000-1,005$ & 0,198 \\
\hline & $\leq 30$ dias vs. $>30$ dias* & 0,15 & $0,03-0,74$ & 0,012 \\
\hline Existência de cuidador* & & 0,47 & $0,03-7,83$ & 0,55 \\
\hline \multirow[t]{3}{*}{ Situação laboral do cuidador } & Inativo/desempregado vs. restantes* & 2,09 & $0,71-6,15$ & 0,177 \\
\hline & Empreg. conta própria vs. restantes* & 0,68 & $0,13-3,71$ & 1,000 \\
\hline & Empreg. conta outrem vs. restantes* & 0,51 & $0,16-1,66$ & 0,262 \\
\hline \multirow[t]{3}{*}{ Parentesco do cuidador } & Cônjuge/companheiro vs. restantes* & 0,77 & $0,27-2,22$ & 0,627 \\
\hline & Filho vs. restantes* & 1,24 & $0,44-3,52$ & 0,681 \\
\hline & Irmão vs. restantes* & 1,08 & $0,09-12,56$ & 1,000 \\
\hline Tempo despendido como cuidador ${ }^{\S}$ & & 0,99 & $0,98-1,00$ & 0,098 \\
\hline
\end{tabular}

* Teste $\chi^{2} .{ }^{\S}$ Regressão logística.

Legenda: $\mathrm{OR}=$ Razão de probabilidades, odds ratio; IC = Intervalos de confiança; NUTS = Nomenclatura das unidades territoriais para fins estatísticos; $\mathrm{APU}=$ Área predominantemente urbana; $\mathrm{APR}=$ Área predominantemente rural; $\mathrm{ECSCP}=$ Equipa comunitária de suporte em cuidados paliativos. Nível de significância $p<0,05$.

resultado está de acordo com um estudo que obteve uma associação positiva entre a ocorrência da morte no domicílio e uma referenciação à equipa de cuidados paliativos com antecedência superior a um mês em relação ao óbito. ${ }^{27}$ Torna-se, assim, importante que os profissionais estejam habilitados a reconhecer precocemente a necessidade de cuidados paliativos para um determinado doente. Por outro lado, é essencial que uma rede de cuidados paliativos seja gerida e atue de forma ágil para dar resposta a situações urgentes, nas quais um dia poderá fazer a diferença na forma como decorre a morte.

Ser cuidado numa ECSCP foi também um fator facilitador, aumentando nove vezes a probabilidade de morrer no domicílio entre os doentes que assim o desejavam. Esta influência vai ao encontro de um estudo em que foram identificados como fatores influenciadores o envolvimento de uma equipa especialista em cuidados paliativos e o ser cuidado no domićlio no último mês, entre outros. ${ }^{8}$ Em relação a este aspeto, 
estimando que uma ECSCP deverá dar resposta a 100.000 habitantes, ${ }^{28}$ Portugal necessitaria de pelo menos 103 equipas, o que está ainda muito aquém das 23 equipas registadas em fontes oficiais em fevereiro de $2019 . .^{29}$

Quanto às restantes variáveis relativas ao doente não foram identificadas associações estatisticamente significativas, não sendo de desprezar o baixo número de doentes em algumas categorias.

Finalmente, em relação aos cuidadores, nem a sua existência nem qualquer outra característica estudada revelaram influenciar a satisfação do desejo de morte no domicílio. Relacionado com estes aspetos, seria ainda interessante determinar a preferência dos familiares cuidadores destes doentes e identificar os fatores que a influenciam, já que estes parecem ter uma elevada influência no local de ocorrência da morte. ${ }^{24,30}$

Como principais limitações aponta-se, por um lado, a baixa taxa de resposta global, podendo estar relacionada com o trabalho suplementar solicitado às equipas de cuidados paliativos, um recurso ainda escasso no país e sob pressão assistencial. Por outro lado, a fraca representação de alguns doentes, como os doentes acompanhados por EIHSCP, os doentes não oncológicos e os residentes no Alentejo, no Algarve e no arquipélago da Madeira, contribuindo para tal facto também a assimetria geográfica no que se refere à cobertura em cuidados paliativos especializados. Estas limitações tornaram impossível a extrapolação dos resultados para o contexto nacional, tendo em conta uma amostra não representativa pela sua dimensão e pelas suas características.

Abordando a validade interna do estudo destaca-se, desde logo, a perda de $82 \%$ de doentes, em relação à amostra do estudo global do OPCP, por estes não desejarem morrer no domicílio e/ou por não apresentarem local de morte registado. Salienta-se também a existência de dados omissos, principalmente na avaliação do bem-estar funcional, sendo incluídos na análise desta variável apenas onze doentes. Como vieses, aponta-se ainda o possível viés de aferição na aplicação do FACIT-Pal.

Sugere-se que sejam realizados novos estudos no futuro com estratégias que ampliem a taxa de resposta, de forma a determinar com maior precisão as preferências dos doentes portugueses, o local onde morrem e o grau de concordância. Estudos com maior amostra poderão também confirmar as associações aqui identificadas, assim como testar associações com fatores identificados na literatura como influenciadores, mas sem associação estatisticamente significativa no presente estudo.

No futuro seria interessante determinar também o local de morte menos desejado por estes doentes, uma vez que uma possível estratégia poderá ser evitar que o doente morra nesse local, já que muitas vezes pode não ser possível respeitar o local preferido por circunstâncias onde o núcleo doente-família-equipa não consegue intervir.

\section{CONCLUSÃO}

No presente estudo a concordância entre a preferência e a realidade foi apenas razoável, coincidindo aproximadamente em $1 / 3$ dos doentes.

Identificou-se que a referenciação e/ou admissão atempadas às equipas prestadoras de cuidados paliativos poderão ter um papel importante. Verificou-se também que a ECSCP poderá ser uma das áreas de atuação no sentido do aumento da satisfação das preferências. Os resultados obtidos alertam ainda para possíveis desigualdades entre os diferentes locais de residência dos doentes.

Os fatores facilitadores identificados no presente estudo deverão auxiliar e orientar a importante tarefa que é o desenvolvimento dos cuidados paliativos, nomeadamente ao nível dos cuidados de saúde primários, contribuindo possivelmente para travar a tendência prevista e insustentável de um decréscimo de morte domiciliar nos próximos anos em Portugal.

\section{REFERÊNCIAS BIBLIOGRÁFICAS}

1. Tang ST. When death is imminent: where terminally ill patients with cancer prefer to die and why. Cancer Nurs. 2003;26(3):245-51.

2. Sadler E, Hales B, Henry B, Xiong W, Myers J, Wynnychuk L, et al. Factors affecting family satisfaction with inpatient end-of-life care. PLoS One. 2014;9(11):e110860.

3. Sapeta AP, Capelas ML, Ferreira RC, Batista S, Pereira SM. Relatório Outono 2018 [homepage]. Lisboa: Universidade Católica Editora; 2020. Available from: https://www.uceditora.ucp.pt/site/custom/template/ucptpl_uce.asp?SSPAGEID=2746\&lang=1\&artigoID=36879

4. Gomes B, Sarmento VP, Ferreira PL, Higginson IJ. Estudo epidemiológico dos locais de morte em Portugal em 2010 e comparação com as preferências da população portuguesa [Epidemiological study of place of death in Portugal in 2010 and comparison with the preferences 
of the Portuguese population].Acta Med Port. 2013;26(4):327-34. Portuguese

5. Capelas ML, Coelho SP. Local de prestação de cuidados no final da vida e local de morte: preferências dos portugueses [Place of care at the end-of-life and to die: portuguese people's preferences]. Cad Saúde. 2013;6:7-18. Portuguese

6. Instituto Nacional de Estatística. Estatísticas da saúde 2015 [homepage]. Lisboa: INE; 2017. Available from: https://www.ine.pt/xportal/ xmain? xpid=INE\&xpgid=ine_publicacoes\&PUBLICACOESpub_boui $=2$ 57779974\&PUBLICACOESmodo $=2$

7. Sarmento VP, Higginson IJ, Ferreira PL, Gomes B. Past trends and projections of hospital deaths to inform the integration of palliative care in one of the most ageing countries in the world. Palliat Med. 2016;30 (4):363-73.

8. Burge F, Lawson B, Johnston G, Asada Y, McIntyre PF, Flowerdew G. Preferred and actual location of death: what factors enable a preferred home death? J Palliat Med. 2015;18(12):1054-9.

9. Raijmakers NJ, de Veer AJ, Zwaan R, Hofstede JM, Francke AL. Which patients die in their preferred place? A secondary analysis of questionnaire data from bereaved relatives. Palliat Med. 2018;32(2): 347-56.

10. Bannon F, Cairnduff V, Fitzpatrick D, Blaney J, Gomes B, Gavin A, et al. Insights into the factors associated with achieving the preference of home death in terminal cancer: a national population-based study. Palliat Support Care. 2018;16(6):749-55.

11. Meeussen K, Van Den Block L, Bossuyt N, Bilsen J, Echteld M, Van Casteren $\mathrm{V}$, et al. GPs' awareness of patients' preference for place of death. Br J Gen Pract. 2009;59(566):665-70.

12. Capelas ML, Ferreira PL, Sapeta P, Gomes B, Pereira S, Simões C, et al. Cuidados paliativos em Portugal: caracterização de doentes referenciados e admitidos, equipas, avaliação de indicadores de qualidade, efetividade do controlo sintomático e local de morte [Internet]. Lisboa: [s.n.]; 2016 [cited 2017 Apr 21].Available from: https://ics.lisboa.ucp.pt/ asset/3406/file

13. Instituto Nacional de Estatística. Divisão administrativa [homepage]. Lisboa: INE; [s.d.] [cited 2018 Mar 20]. Available from: https:// www.ine.pt/xportal/xmain?xpid=INE\&xpgid=ine_cont_inst\&INST=62 51013

14. Lyons KD, Bakitas M, Hegel MT, Hanscom B, Hull J, Ahles TA. Reliability and validity of the Functional Assessment of Chronic Illness Therapy-Palliative care (FACIT-Pal) scale. J Pain Symptom Manage. 2009;37 (1):23-32.

15. FACIT Group. Functional assessment of chronic illness therapy: palliative care [homepage]. FACIT Group; 2020 [cited 2021 Jan 31]. Available from: https://www.facit.org/measures/FACIT-Pal

16. Higginson IJ, Daveson BA, Morrison RS, Yi D, Meier D, Smith M, et al. Social and clinical determinants of preferences and their achievement at the end of life: prospective cohort study of older adults receiving palliative care in three countries. BMC Geriatr. 2017;17(1):271.

17. Connor SR, Bermedo MS. Global atlas of palliative care at the end of life [Internet]. London:Worldwide Palliative Care Alliance; 2014 [cited 2017 Apr 2]. Available from: https://www.who.int/nmh/Global_Atlas_ of_Palliative_Care.pdf
18. Townsend J, Frank AO, Fermont D, Dyer S, Karran O, Walgrove A, et al. Terminal cancer care and patients' preference for place of death: a prospective study. BMJ. 1990;301(6749):415-7.

19. Brogaard T, Neergaard MA, Sokolowski I, Olesen F, Jensen AB. Congruence between preferred and actual place of care and death among Danish cancer patients. Palliat Med. 2013;27(2):155-64.

20. Howell DA, Wang HI, Roman E, Smith AG, Patmore R, Johnson MJ, et al. Preferred and actual place of death in haematological malignancy. BMJ Support Palliat Care. 2017;7(2):150-7.

21. Thomas C, Morris SM, Clark D. Place of death: preferences among cancer patients and their carers. Soc Sci Med. 2004;58(12):2431-44.

22. Agar M, Currow DC, Shelby-James TM, Plummer J, Sanderson C, Abernethy AP. Preference for place of care and place of death in palliative care: are these different questions? Palliat Med. 2008;22(7):787-95.

23. Beccaro M, Costantini M, Rossi PG, Miccinesi G, Grimaldi M, Bruzzi P. Actual and preferred place of death of cancer patients: results from the Italian survey of the dying of cancer (ISDOC). J Epidemiol Community Health. 2006;60(5):412-6.

24. Gomes B, Higginson IJ. Factors influencing death at home in terminally ill patients with cancer: systematic review. BMJ. 2006;332(7540):51521.

25. Gomes B, Higginson IJ, Calanzani N, Cohen J, Deliens L, Daveson BA, et al. Preferences for place of death if faced with advanced cancer: a population survey in England, Flanders, Germany, Italy, The Netherlands, Portugal and Spain. Ann Oncol. 2012;23(8):2006-15.

26. Gu X, Cheng W, Cheng M, Liu M, Zhang Z. The preference of place of death and its predictors among terminally ill patients with cancer and their caregivers in China. Am J Hosp Palliat Med. 2015;32(8):835-40.

27. Poulose JV, Do YK, Neo PS. Association between referral-to-death interval and location of death of patients referred to a hospital-based specialist palliative care service. J Pain Symptom Manage. 2013;46(2): 173-81.

28. Radbruch L, Payne S. White Paper on standards and norms for hospice and palliative care in Europe: part 2. Eur J Palliat Care. 2010;17(1): 22-33.

29. Serviço Nacional de Saúde. Equipas de cuidados paliativos [homepage]. Lisboa: SNS; 2019 [updated 2021 Jan 7; cited 2019 Apr 2]. Available from: https://www.sns.gov.pt/sns/cuidados-paliativos/unidades-decuidados-paliativos/

30. Ikezaki S, Ikegami N. Predictors of dying at home for patients receiving nursing services in Japan: a retrospective study comparing cancer and non-cancer deaths. BMC Palliat Care. 2011;10:3.

\section{CONFLITO DE INTERESSES}

Os autores declaram não possuir quaisquer conflitos de interesse.

\section{ENDEREÇO PARA CORRESPONDÊNCIA}

Rita Carvalho Cunha Ferreira

E-mail: ritafccferreira@netcabo.pt

https://orcid.org/0000-0003-0715-1752

Recebido em 24-10-2019

Aceite para publicação em 10-03-2021 


\section{ABSTRACT}

\section{DYING AT HOME: FACTORS RELATED TO SATISFYING PATIENT PREFERENCE}

Background: Place of death has been studied and acknowledged as relevant in palliative care. In Portugal, it seems that most patients prefer to die at home, although that preference is not satisfied.

Aim: Identify the factors related to satisfying patients' preference to die at home among palliative care recipients in Portugal. Methods: An epidemiologic, observational, cross-sectional, and analytic study was performed in patients treated during 2017 in palliative care facilities that answered a research work conducted by the Portuguese Observatory for Palliative Care. A set of independent variables concerning demographic, social, clinical, and healthcare characterization was selected. Chi-Square and Mann-Whitney tests and a binary logistic regression model were employed for the bivariate statistical analysis. The multivariate analysis was performed by using a logistic regression model based on the Forward method considering the Wald test.

Results: We obtained a 9.8\% response rate from the institutions, which included 68 patients. $52.9 \%$ were male and the median age was 78.5 (IQR=19). The preference to die at home was met in $32.3 \%(k=0.26)$. We found statistically significant associations with residence in the Azores $(O R=3.79)$, and in rural areas $(O R=10.00)$, duration of follow up in a palliative care team ( $\leq 30$ days, $O R=0.15)$, and assistance by home palliative care teams $(\mathrm{OR}=9.00)$.

Conclusions: We obtained a fair satisfaction degree of patients' preference to die at home, with one of the lowest values observed in the literature. We identified an association with place of residence, duration of follow-up in palliative care, and assistance by home specialized teams. These enabling factors agree with those previously reported in other international studies. They should guide strategies definition adjusted to the reality of the Portuguese population.

Keywords: Palliative care; Terminal care; Patient preferences; Place of death; Home death. 